Cahiers d'études africaines

\title{
Chalaye, Sylvie. - Nègres en images
}

Préface de Blaise Ndjehoya, L'Harmattan ("La Bibliothèque

d'Africultures »), 2002, 191 p., bibliographie.

Jean-Paul Colleyn

\section{(2) OpenEdition}

\section{Journals}

Édition électronique

URL : http://journals.openedition.org/etudesafricaines/4693

DOI : 10.4000/etudesafricaines.4693

ISSN : $1777-5353$

Éditeur

Éditions de l'EHESS

\section{Édition imprimée}

Date de publication : 1 janvier 2004

Pagination : 453-455

ISBN : 978-2-7132-1823-1

ISSN : 0008-0055

Référence électronique

Jean-Paul Colleyn, «Chalaye, Sylvie. - Nègres en images », Cahiers d'études africaines [En ligne],

173-174 | 2004, mis en ligne le 08 mars 2007, consulté le 21 septembre 2020. URL : http://

journals.openedition.org/etudesafricaines/4693; DOI : https://doi.org/10.4000/etudesafricaines.4693

Ce document a été généré automatiquement le 21 septembre 2020.

(c) Cahiers d'Études africaines 


\title{
Chalaye, Sylvie. - Nègres en images
}

\author{
Préface de Blaise Ndjehoya, L'Harmattan («La Bibliothèque \\ d'Africultures »), 2002, 191 p., bibliographie.
}

Jean-Paul Colleyn

1 Dans ce livre élaboré à partir d'articles parus dans diverses revues, Sylvie Chalaye, maître de conférences en études théâtrales à l'Université de Rennes-II, se livre à une sorte d'archéologie du racisme, triomphant ou rampant selon les époques, dans la culture française. Elle retrace, comme le dit son préfacier, au long de cinq siècles, la généalogie de l'invisibilité des Noirs dans les représentations françaises. La France, décidément, ne veut pas se remettre de s'être livrée à la "traite des Noirs ", ni d'avoir mené l'entreprise coloniale sans état d'âme. L'auteure aborde différents domaines de la culture mais privilégie le théâtre, non seulement parce que c'est sa spécialité, mais aussi parce que le théâtre est resté longtemps le seul vrai média populaire, instrument d'information et de vulgarisation.

Dans ses deux premiers tiers, le livre retrace un parcours historique, dont la lecture, en raison de la forme de recueil, ne peut se faire qu'en dents de scie, avec de fréquentes avancées et de fréquents reculs sur l'échelle du temps. Mais le lecteur s'y habitue et ne peut manquer d'être captivé par la richesse de la documentation et l'intérêt d'une analyse du «traitement» de l'image des Antillais et des Africains dans les arts du spectacle français. Si, pour les mêmes raisons de structure, résumer l'ouvrage est une entreprise malaisée, il paraît utile de donner quelques points de repères pour suggérer l'ampleur d'un vaste passage en revue bien documenté, utile et édifiant.

3 Tout l'art du xvii ${ }^{e}$ siècle s'emploie à ignorer la nature esclavagiste du négoce qui enrichit les armateurs. Au siècle des Lumières, les Noirs sont plus présents et inspirent les écrivains, mais leur image oscille entre la laideur et le phantasme sexuel. Ceux qui tiennent la vedette, dans la littérature comme dans la peinture, ce sont les pages, négrillons et domestiques qui égayent les assemblées mondaines. À la fin du siècle, un décret lui dénie le statut de citoyen en interdisant aux prêtres et aux notaires de célébrer des mariages mixtes. En revanche, de nombreuses pièces de l'époque révolutionnaire mettent en scène la confraternité de tous les hommes quelle que soit la couleur de leur peau. Mais si les philosophes dénoncent les abus de l'esclavage, la traite 
elle-même n'est jamais condamnée ; dans le théâtre et la peinture révolutionnaires, la représentation $\mathrm{du}$ travail des esclaves sur les plantations continue à être soigneusement évitée. La République invente le modèle du bon nègre reconnaissant et fidèle, capable de se sacrifier pour son maître et sans projet «personnel ». Le premier héros noir $\mathrm{du}$ théâtre français devait être un nègre affranchi. Après la révolution, qui avait amené la libération des théâtres, on vit fleurir des pièces anti-esclavagistes, mais le héros reste le Blanc abolitionniste, le bon Noir restant confiné au rôle de sujet reconnaissant envers la République salvatrice. Aucune pièce ne met en scène les conditions de travail des esclaves, un sujet qui demeure tabou. La première figure du "nègre tragique", un héros révolutionnaire qui annonce de manière prémonitoire Toussaint Louverture, est refusée par le public et la critique. Elle apparaît en 1791, sous les traits de Télémaque, dans la pièce Le Blanc et le Noir, de Charles-Antoine PigaultLebrun, mais la pièce est retirée de l'affiche après la troisième représentation. La figure d'Adonis ou le bon nègre, lui est préférée : Adonis trahit la confiance de Biassou, un chef sanguinaire à la tête d'une armée de nègres révoltés, pour sauver ses bienveillants maitres qui, finalement, distribuent les récompenses. En étudiant les échecs de certains sujets, Sylvie Chalaye montre, à partir des extraits de presse de l'époque, que le héros ou l'héroïne noire demeure pendant longtemps à ce point l'Autre qu'aucun spectateur ne saurait s'y identifier. Ce sont les romantiques qui osèrent, les premiers, faire monter sur la scène française des héros noirs, non sans démêlés avec la censure. Sous l'Empire, alors que Napoléon essuyait des échecs cuisants contre Toussaint Louverture et qu'il rétablissait l'esclavage, les Caraïbes devenaient un sujet proscrit au théâtre. Sous la restauration, les auteurs ont dû accepter les compromis qu'exigeait d'eux la police littéraire "afin de donner du nègre l'image qui convenait» (p. 80). Les romantiques heurtèrent les préjugés coloniaux, les pièces de Victor Hugo et d'Alfred de Vigny furent censurées et vilipendées par la critique. Après la révolution de juillet, sous LouisPhilippe, le lobby colonial perdit sont influence, certains tabous sont levés, mais le nègre métissé par l'esclavage devient le «monstre le plus sanguinaire de la scène française ", monstre de souffrance et de cruauté, engendré par l'avidité et le luxe. Victime, le nègre provoque la pitié, libre, il inspire la peur. Une fois les sujets " coloniaux » autorisés, se répand une vogue de ce que l'on a appelé, à l'époque, le "mélano-drame", avec comme personnage récurent le sang-mêlé presque blanc, qui ignore ses origines ou préfère les cacher. Si la scène romantique avait fait progresser l'opinion publique en dénonçant les préjugés de couleur, l'Europe allait s'inventer de nouveaux arguments pour se lancer dans la colonisation de l'Afrique. En 1892, après la victoire de l'armée française au Dahomey, la plupart des théâtres programmèrent des pièces à grand spectacle qui mettaient en scène de sauvages guerriers africains que les courageux soldats français parvenaient à mater. On exhibe une Afrique sauvage pour justifier la «mission civilisatrice » : le Soudan aux Arènes du bois de Boulogne, le Congo à l'hippodrome, les revues exotiques au music-hall (Zoulous aux Folies-Bergère, Dahoméens et Amazones, au casino). Les reconstitutions pseudo ethnographiques prennent le relais : les Achantis (1887) puis les Somalis (1895) au Jardin d'Acclimatation, un village d'Abyssinie à l'hippodrome (1895). Au début du xx siècle, le théâtre du Grand Guignol multiplie les pièces (notamment de Charles Dullin, Henri-René Lenormand, André-Paul Antoine) qui puisent l'essentiel de leurs effets dans la peur que suscitaient les indigènes d'Afrique et leurs coutumes sanglantes. Mais le bon produit de la colonie, le tirailleur, domestiqué, fort, bonhomme et loyal, fait également partie des stéréotypes, tant au théâtre que dans les chansons; une image renforcée par le rôle 
qu'ils jouèrent pendant la guerre 14-18. C'est après l'armistice que le caricatural « Y-abon » des affiches Banania devient un personnage familier.

Dans les années 1920, la mise en scène du corps noir du danseur acteur Habib Benglia, dont la critique salue la grâce animale, « illustre bien la place nouvelle que l'on donnera bientôt au nègre ». Elle consiste à incarner l'âme primitive, toute sensitive et charnelle. Le nègre est exploité " comme imago du ça ", même si ce procédé concourt à dénoncer les perversités de l'Occident.

5 L'analyse des Nègres, de Jean Genet, pièce iconoclaste qui n'a rien perdu, hélas, de sa force corrosive, sert de charnière au livre. Sylvie Chalaye crédite, à juste titre, Jean Genet d'avoir, par le théâtre, dénoncé combien l'aliénation du nègre est une question dramatique au premier sens du terme puisqu'elle résulte de la mise en scène des Noirs par les Blancs, tout au long des siècles marqués par l'esclavage et la colonisation. Suit une critique de La tragédie du Roi Christophe d'Aimé Césaire monté par Jacques Nichet en 1996-1997. Nichet a voulu faire de Christophe la figure emblématique et universelle du libérateur devenu lui-même dictateur, alors que, d'après Chalaye, Césaire, qui a écrit cette pièce dans la mouvance du mouvement de la négritude, dénonce en Christophe la tragique méprise du nègre qui redresse fièrement la tête mais continue de voir le monde à travers les yeux du Blanc.

6 Les dernières pages du livre traitent de la période actuelle, marquée, malgré l'effort de distribution planétaire d'un Peter Brook aux Bouffes du Nord, par la rareté des acteurs noirs dans le paysage artistique français. Dans un entretien, l'acteur guadeloupéen Greg Germain remarque que, brusquement après 1985, malgré l'explosion audiovisuelle, les acteurs noirs disparaissent de la télévision, si ce n'est deux ou trois dans des rôles subalternes. Tout se passe comme si les responsables avaient intériorisé le fait qu'une partie de la France étant raciste, pour garder de bons scores d'audience, il faut proposer des images qui ne dérangent personne. Sous-jacent à ces dernières pages se pose le débat sur le bien-fondé des quotas pour corriger l'ostracisme audiovisuel dont sont victimes les minorités «de couleur» dans la patrie des droits de l'homme. Ce débat, lancé par le collectif Égalité, suscite des positions diverses: Greg Germain pour, Marco Prince, star de la musique passé acteur, contre.

7 Au terme de ce qui, sous un faible volume, apparaît comme une somme, qui n'est pas sans rappeler, dans son esprit quoiqu'en des dimensions plus modestes, L'orientalisme d'Edward Said ou L'Invention de l'Afrique de V. Y. Mudimbe, on saluera l'utilité de ce travail, dont la bibliographie peut, par ailleurs, se révéler précieuse. 